\title{
Intravitreal ranibizumab alone or in combination with Calcium dobesilate (CaD) for the treatment of diabetic macular edema in non-proliferative diabetic retinopathy patients: a 12-month outcomes of a retrospective study
}

Dongxuan Wang

Changyi People's Hospital

Hui Wang

Changyi People's Hospital

Shuang Wu ( $\nabla$ cyrmyyyk1234@126.com )

Changyi People's Hospital

Xueqiu Yang

Changyi People's Hospital

Jiansen Xu

Changyi People's Hospital

\section{Research Article}

Keywords: Calcium dobesilate, Diabetic macular edema, Ranibizumab

Posted Date: March 7th, 2022

DOI: https://doi.org/10.21203/rs.3.rs-1086413/v1

License: (c) (1) This work is licensed under a Creative Commons Attribution 4.0 International License.

Read Full License 


\section{Abstract}

Purpose To investigate the effect of calcium dobesilate $(\mathrm{CaD})$ in the treatment of diabetic macular edema (DME) in patients with non-proliferative diabetic retinopathy (NPDR).

Methods This retrospective, observational, consecutive case control study enrolled patients newly diagnosed with DME who received intravitreal ranibizumab (IVR) administration with 3-monthly loading dose injection followed by pro re nata (3+PRN) regimen with or without $\mathrm{CaD}$ orally daily for at least 12 month follow-up. Medical records and optical coherence tomography (OCT) results were reviewed and compared.

Results A total of 102 eyes from 102 patients were enrolled in this study. Fifty-four patients received IVR combined with CaD orally (IVR+CaD group), while forty-eight patients received IVR solely (IVR group). At every follow-up, 3, 6 and 12 months after injection, the best corrected visual acuity (BCVA) improved and the central macular thickness (CMT) decreased in both groups when compared with those at baseline ( $P$ $<0.05$ ), while there were no significant differences in BCVA improvement and CMT reduction between the two groups $(P>0.05)$. The mean number of ranibizumab injections in $R+C$ group was significantly lower than that in $\mathrm{R}$ group $(5.4 \pm 1.1$ injections versus $6.7 \pm 1.6$ injections, $\mathrm{P}<0.05)$ within 1 -year treatment. No adverse events were found in neither groups.

Conclusions Adding orally $\mathrm{CaD}$ to intravitreal ranibizumab was demonstrated to have similar effectiveness and safety for improving visual function and restoring the anatomy of the retina in macular with fewer injections in DME patients.

\section{Introduction}

Diabetic macular edema (DME), characterised by exudative fluid accumulation in the macula, is the leading cause of visual impairment in patients with diabetes mellitus (DM)[1,2]. The blood retinal barrier (BRB) is vital for maintaining the normal structure and function of the retina, and DME is caused by its failure[3]. The disruption of BRB is associated with an increase in the level of vascular endothelial growth factor (VEGF), intercellular adhesion molecule-1 (ICAM-1), interleukin-6 (IL-6), monocyte chemotactic protein-1 (MCP-1), and other inflammatory factors[3]. Therefore, anti-VEGF[4, 5] and other antiinflammatory agents[6] become the main treatment way to deal with DME after laser photocoagulation. Although intravitreous aflibercept, bevacizumab, or ranibizumab had been proved to improve vision function in eyes with center-involved DME in a comparative effectiveness randomized clinical trial conducted by Diabetic Retinopathy Clinical Research Network [7], there were still a significant number of patients with poor response to anti -VEGF treatment or without sustained improvement in visual acuity. Although VEGF inhibitor therapy had been shown to be a cost-effective treatment for DME that had reduced the incidence of vision loss and could reduce the associated economic and social cost[8], continuous intravitreal injection treatment also brings a high economic burden to patients, especially in developing countries. Therefore, intravitreal anti-VEGF agents in combined with other therapy, such as 
intravitreal triamcinolone acetonide[9], or Rho-kinase inhibitor[10], or methotrexate[11], subthreshold micropulse laser[12, 13], macular grid laser photocoagulation[14], sub-tenon steroid injection[15], or switching anti-VEGF treatment to intravitreal dexamethasone implant[6, 16-18], was investigated in DME patients.

Calcium dobesilate $(\mathrm{CaD})$ is a vascular protective drug that has been used in DR treatment in past decades[19-21] by its effect in preventing the oxidative stress and inflammation[22]. It can protect blood vessels and improve circulation by lowering blood viscosity, inhibiting platelet activity and reducing capillary permeability $[23,24]$. Nonetheless, the potential beneficial effects of CaD on DME patients when combined with intravitreal anti-VEGF agents was still not clear. Therefore, in this study, we investigated the effectiveness and safety of combined therapy of CaD with anti-VEGF agents for the treatment of DME in NPDR patients. The primary outcome of this study was BCVA and CMT changes after the treatment and the second outcome was the injection numbers of ranibizumab administered during the follow-up period.

\section{Methods}

\section{Patient selection}

A retrospective, observational, case control study was conducted on patients with treatment-naive centerinvolved DME (CIDME) in NPDR patients, who received intravitreal ranibizumab treatment in the department of ophthalmology, Changyi Peoplie's Hospital with one-year follow-up period. Patients with type 2 diabetes mellitus and clinically significant DME, according to the criteria developed by ETDRS[25] were included in the study, and the flowchart (Figure 1) indicated the protocol to select the patients.

This study adhered to the tenets of the Declaration of Helsinki and was approved by the Ethics Committee of Changyi People's Hospital. Informed consent was obtained from all of the patients or their guardians before initiation of intravitreal pharmacotherapy. The medical records were retrospectively reviewed after approval was received from the Institutional Review Board, Changyi People's Hospital.

\section{Data collection}

The clinical data retrieved from medical records included demographic information, general medical history, history of any previous ocular conditions, previous treatments for DME. All participants underwent a detailed ophthalmologic examination, including best corrected visual acuity (BCVA), slit-lamp biomicroscopy, intraocular pressure measurement, fundus examination under pupil dilation, and macular scan by SD-OCT (Cirrus HD-OCT with software version 6.0; Carl Zeiss Meditec, Dublin, CA, USA), before the treatment and at each follow-up. The central macular thickness (CMT) was defined as the retinal thickness of the central $1.0 \mathrm{~mm}$ at OCT B-scan.

In addition, the occurrence of any complications during the perioperative period and postoperative followup were also analyzed for safety evaluation. 


\section{Treatment protocol}

A 3+PRN regimen was used in this study. After 3-monthly loading dose injection of intravitreal ranibizumab (IVR), patients received re-injection if needed. The criteria for re-injection was similar as described previously[26]. At each visit, patients underwent visual acuity assessment and OCT scan for macular thickness assessment. If BCVA and OCT were stable for 2 consecutive visits, reinjection was suspended, while if worsening of BCVA ( $>5$ letter loss) or OCT (increase $>10 \%$ ) was noticed at follow-up, reinjection was given.

In IVR group, patients received IVR injection only as mentioned above, while in IVR+CaD group, patients received capsules of $\mathrm{CaD}(1500 \mathrm{mg}$ per day in three divided doses of $500 \mathrm{mg}$ ) besides IVR injection.

\section{Statistical analysis}

All data were analyzed using the SPSS (version 11.5, SPSS Inc., Chicago, IL, USA). BCVA measurements were converted to logMAR equivalents for statistical analysis. Pearson chi-square test was used for comparative analyses of categorical variables. The independent sample t-test and paired t-test were employed to analyze changes in BCVA and CFT. For all statistical tests, $p<0.05$ was considered significant.

\section{Results}

Between January 2017 and March 2020, 102 patients (mean age of $57.72 \pm 9.91$, rang of 42-69 years) were enrolled in the present study, which 63 patients $(61.76 \%)$ were male and 39 patients $(38.24 \%)$ were female. Fifty-four patients, who received IVR combined with CaD orally, were referred as IVR+CaD group, while forty-eight patients, who received IVR solely, were referred as IVR group.

Table 1 shows the baseline characteristics for the study population. There was no significant difference between the two groups in terms of gender, age, history of smoking and drinking, other comorbidities except diabetes, the course of diabetes and HbA1c. 
Table 1

Baseline characteristics of total study population

\begin{tabular}{|llll|}
\hline & IVR $(\mathbf{n}=\mathbf{4 8})$ & IVR+CaD $(\mathbf{n}=\mathbf{5 4})$ & $\mathbf{p}$ value \\
\hline gender (male/female) & $31 / 17$ & $32 / 22$ & 0.581 \\
\hline age (years) & $56.98 \pm 11.62$ & $57.65 \pm 12.79$ & 0.784 \\
\hline smoking (yes/no) & $28 / 20$ & $29 / 25$ & 0.638 \\
\hline drinking (yes/no) & $19 / 29$ & $25 / 29$ & 0.494 \\
\hline comorbidity (yes/no) & $17 / 31$ & $18 / 36$ & 0.825 \\
\hline course (years) & $10.58 \pm 6.00$ & $11.51 \pm 6.16$ & 0.440 \\
\hline HbA1c (\%) & $7.72 \pm 0.88$ & $7.80 \pm 1.01$ & 0.645 \\
\hline
\end{tabular}

As shown by Table 2, after IVR administration with 3-monthly loading dose injection, the BCVA (logMAR) significantly increased from $0.35 \pm 0.12$ to $0.24 \pm 0.09$ (pष0.05) in IVR group and increased from $0.32 \pm 0.15$ to $0.23 \pm 0.10(p \otimes 0.05)$ in IVR+CaD group, and the BCVA maintained satisfactory during the treatment until the end of the follow-up in both groups. Meantime, the CMT significantly decreased from $427 \pm 172 \mu \mathrm{m}$ to $286 \pm 69 \mu \mathrm{m}(\mathrm{p} \otimes 0.05)$ in IVR group and decreased from $412 \pm 185 \mu \mathrm{m}$ to $277 \pm 92 \mu \mathrm{m}$ (pष0.05) in IVR+CaD group, and the CMT also maintained during the treatment until the end of the follow-up in both groups. No significant difference was found in BCVA and CMT between two groups at baseline and every followup period.

Table 2

Changes of CMT and BCVA after the treatment

\begin{tabular}{|c|c|c|c|c|c|c|}
\hline & \multicolumn{3}{|c|}{ BCVA (logMAR) } & \multicolumn{3}{|l|}{ CMT $(\mu \mathrm{m})$} \\
\hline & IVR $(n=48)$ & $\begin{array}{l}\text { IVR+CaD } \\
(n=54)\end{array}$ & $\begin{array}{l}\mathrm{p} \\
\text { value }\end{array}$ & IVR $(n=48)$ & $\begin{array}{l}\text { IVR+CaD } \\
(n=54)\end{array}$ & $\begin{array}{l}\mathrm{p} \\
\text { value }\end{array}$ \\
\hline Baseline & $0.35 \pm 0.04$ & $0.34 \pm 0.04$ & 0.416 & $428.21 \pm 100.68$ & $424.48 \pm 88.42$ & 0.843 \\
\hline At 3 month & $0.24 \pm 0.04^{*}$ & $0.23 \pm 0.04^{\star}$ & 0.253 & $288.27 \pm 19.83^{*}$ & $282.06 \pm 26.55^{\star}$ & 0.188 \\
\hline At 6 month & $0.26 \pm 0.04^{*}$ & $0.25 \pm 0.04^{*}$ & 0.436 & $281.40 \pm 27.34^{*}$ & $274.33 \pm 31.74^{*}$ & 0.234 \\
\hline $\begin{array}{l}\text { At } 12 \\
\text { month }\end{array}$ & $0.25 \pm 0.03^{*}$ & $0.24 \pm 0.04^{*}$ & 0.696 & $269.33 \pm 28.82 *$ & $264.93 \pm 27.16^{*}$ & 0.429 \\
\hline
\end{tabular}

Within one year of IVR treatment by $3+P R N$ regimen, the mean injection number in IVR group was $6.7 \pm$ 1.6 , which was significantly more than that in IVR+CaD group $(5.4 \pm 1.1, \mathrm{p} \otimes 0.05)$. 
As for complications during the treatment, there were no ocular complications, such as endophthalmitis, vitreous hemorrhage, and retinal detachment, and no serious systemic adverse events such as cerebral infarction or myocardial infarction in neither groups.

\section{Discussion}

DME, also considered as diabetic maculopathy, is one of the most important causes of vision loss among people with diabetes around the world[2]. Although the advent of anti-VEGF agents has greatly improved the treatment effect of DME patients, and intravitreal injection of anti-VEGF agents has become the first line therapy for $\mathrm{DME}$, there are still some patients who response poorly to the treatment.

$\mathrm{CaD}$ (calcium 2,5-dihydroxybenzenesulfonate) is a vascular protective drug which has been proved to have beneficial effects in vascular diseases such as chronic venous insufficiency and haemorrhoids[27] and is extensively used to treat diabetic retinopathy[28-30], choronic venous insufficiency[31] and multiple microangiopathic diseases[32]. In previous studies, the potential mechanisms of CaD improving microcirculation and reducing micro-vascular injury had been investigated as follows[21]: (1) reducing platelet aggregation caused by thrombin or collagen[33]; (2) significantly protecting peritoneal vascular from reactive oxygen species (ROS) effects of penetration[34]; (3) inhibiting capillary permeability[35]; (4) alleviating the chronic inflammatory state and improving endothelial cell function[36, 37]; (5) reducing endothelial shedding by the synthesis and release of nitric oxide (NO)[38]; (6) reducing platelet aggregation by inhibiting prostaglandin and reduce erythrocyte aggregation and viscosity of suspension[24]; (7) down-regulating the expression of VEGF and fibroblast growth factor (FGF) to inhibit vascular endothelial cell proliferation[39]; (8) restoring autophagy by inhibiting the VEGF/PI3K/AKT/mTOR signaling pathway[40]. Therefore, based on these mechanisms mentioned above, $\mathrm{CaD}$ should be effective in the treatment of macular edema. However, in previous studies on the treatment of DME, CaD was found to show neither effect on reducing the risk of development of DME[27], nor statistically significant difference when compared with placebo in respect to combining with laser photocoagulation on the macular thickness in DME patients[41]. These results made it controversial for the role of $\mathrm{CaD}$ in the treatment of macular edema. When we reviewed the literatures about the treatment of $\mathrm{CaD}$ for $\mathrm{DME}$, we found that both the two studies were published relatively early, which were earlier than the wide application of anti-VEGF agents in clinic, and no studies had explored the effectiveness and safety of oral $\mathrm{CaD}$ combined with anti-VEGF treatment. Therefore, our findings, for the first time, showed that compared with anti-VEGF monotherapy, CaD combined with anti-VEGF agents had the similar effect in improving visual function and reducing the CMT in DME patients, and it could reduce the number of intravitreal injection and decrease the economic burden during the 1-year follow-up period.

So far, although the exact mechanism of DME is still unclear, abnormally increased levels of VEGF and inflammatory mediators, and subsequently the breakdown of BRB was considered to play vital role in the formation of macular edema in diabetes[2]. Accordingly, with the emergence of anti-VEGF and antiinflammatory agents, they gradually became the first-line treatment $[42,43]$ or treatment with good prospects $[44,45]$. The effect of CaD on down-regulating VEGF and inhibiting VEGF-related pathways 
might have a synergistic effect with the inhibition of VEGF by anti-VEGF agents[46]. This might partly explain why oral $\mathrm{CaD}$ alone has no therapeutic effect on $\mathrm{DME}$, but it can reduce the number of intravitreal injections when combined with anti-VEGF agents within 1-year follow-up period. In addition, this kind of treatment combination was safe proved by the present study. Neither systemic, nor local complication was found during the treatment. The result showed that although $\mathrm{CaD}$ inhibited the expression of VEGF by cooperating with anti-VEGF agents, and therefore prolonged the treatment duration of anti-VEGF agents, but it did not increase the risk of treatment secondary to VEGF inhibition. This might explain why the application of $\mathrm{CaD}$ alone has no effect in the treatment of DME on the other hand.

\section{Conclusion}

In the current study, oral CaD combined with intravitreal ranibizumab was demonstrated to have similar effectiveness and safety as anti-VEGF monotherapy for improving visual function and restoring the anatomy of the retina in macular with fewer injections in DME patients. Adding oral CaD might be a feasible way to reduce injection number and decrease the economic burden of treatment in DME patients who received anti-VEGF therapy.

\section{Declarations}

\section{Authors' contributions}

Shuang Wu contributed to the ideas and design of study. Dongxuan Wang and Hui Wang performed the study. Xueqiu Yang and Dongxuan Wang collected data, helped in the statistical analysis and drafted the manuscript. Shuang Wu and Jiansen Xu reviewed and revised the manuscript. All authors read and approved the final manuscript.

\section{Availability of data and material}

All data generated or analysed during this study are included in this published article. Data are available at Changyi People's Hospital.

\section{Ethics approval and consent to participate}

This study adhered to the tenets of the Declaration of Helsinki and was approved by the Ethics Committee of Changyi People's Hospital. Informed consent was obtained from all of the patients or their guardians before initiation of intravitreal pharmacotherapy. The medical records were retrospectively reviewed after approval was received from the Institutional Review Board, Changyi People's Hospital.

\section{Consent for publication}

Not applicable.

\section{Conflict of interest}


The authors declare that they have no conflict of interest.

\section{References}

1. Holekamp NM (2016) Overview of diabetic macular edema. Am J Manag Care 22:s284-s291

2. Tan GS, Cheung N, Simo R, Cheung GC, Wong TY (2017) Diabetic macular oedema. Lancet Diabetes Endocrinol 5:143-155

3. Daruich A, Matet A, Moulin A, Kowalczuk L, Nicolas M, Sellam A et al (2018) Mechanisms of macular edema: Beyond the surface. Prog Retin Eye Res 63:20-68

4. Massin P, Bandello F, Garweg JG, Hansen LL, Harding SP, Larsen M et al (2010) Safety and efficacy of ranibizumab in diabetic macular edema (RESOLVE Study): a 12-month, randomized, controlled, double-masked, multicenter phase II study. Diabetes Care 33:2399-2405

5. Akkaya S, Acikalin B, Dogan YE, Coban F (2020) Subthreshold micropulse laser versus intravitreal anti-VEGF for diabetic macular edema patients with relatively better visual acuity. Int $\mathrm{J}$ Ophthalmol 13:1606-1611

6. Rosenblatt A, Udaondo P, Cunha-Vaz J, Sivaprasad S, Bandello F, Lanzetta P et al (2020) A Collaborative Retrospective Study on the Efficacy and Safety of Intravitreal Dexamethasone Implant (Ozurdex) in Patients with Diabetic Macular Edema: The European DME Registry Study. Ophthalmology 127:377-393

7. Diabetic Retinopathy Clinical, Research N, Wells JA, Glassman AR, Ayala AR, Jampol LM, Aiello LP et al (2015) Aflibercept, bevacizumab, or ranibizumab for diabetic macular edema. N Engl J Med 372:1193-1203

8. Hodgson N, Wu F, Zhu J, Wang W, Ferreyra H, Zhang K et al (2016) Economic and Quality of Life Benefits of Anti-VEGF Therapy. Mol Pharm 13:2877-2880

9. Riazi-Esfahani M, Riazi-Esfahani H, Ahmadraji A, Karkhaneh R, Mahmoudi A, Roohipoor R et al (2018) Intravitreal bevacizumab alone or combined with $1 \mathrm{mg}$ triamcinolone in diabetic macular edema: a randomized clinical trial. Int Ophthalmol 38:585-598

10. Ahmadieh H, Nourinia R, Hafezi-Moghadam A, Sabbaghi H, Nakao S, Zandi S et al (2019) Intravitreal injection of a Rho-kinase inhibitor (fasudil) combined with bevacizumab versus bevacizumab monotherapy for diabetic macular oedema: a pilot randomised clinical trial. $\mathrm{Br} \mathrm{J}$ Ophthalmol 103:922-927

11. Fazel F, Oliya B, Mirmohammadkhani M, Fazel M, Yadegarfar G, Pourazizi M (2020) Intravitreal Injections of Bevacizumab Plus Methotrexate versus Bevacizumab Alone for the Treatment of Diabetic Macular Edema: A Randomized, Sham-Controlled Trial. J Curr Ophthalmol 32:164-169

12. Altinel MG, Acikalin B, Alis MG, Demir G, Mutibayraktaroglu KM, Totuk OMG et al (2021) Comparison of the efficacy and safety of anti-VEGF monotherapy versus anti-VEGF therapy combined with subthreshold micropulse laser therapy for diabetic macular edema.Lasers Med Sci10.1007/s10103021-03306-0 
13. Kanar HS, Arsan A, Altun A, Aki SF, Hacisalihoglu A (2020) Can subthreshold micropulse yellow laser treatment change the anti-vascular endothelial growth factor algorithm in diabetic macular edema? A randomized clinical trial. Indian $\mathrm{J}$ Ophthalmol 68:145-151

14. Solaiman KA, Diab MM, Abo-Elenin M (2010) Intravitreal bevacizumab and/or macular photocoagulation as a primary treatment for diffuse diabetic macular edema. Retina 30:1638-1645

15. Eris E, Perente I, Vural E, Vural A, Seymen Z, Celebi ARC et al (2019) Evaluation of the effect of combined intravitreal ranibizumab injection and sub-tenon steroid injection in the treatment of resistant diabetic macular edema. Int Ophthalmol 39:1575-1580

16. Sharma A, Bellala K, Dongre P, Reddy P (2020) Anti-VEGF versus dexamethasone implant (Ozurdex) for the management of Centre involved Diabetic Macular Edema (CiDME): a randomized study. Int Ophthalmol 40:67-72

17. Castro-Navarro V, Cervera-Taulet E, Navarro-Palop C, Monferrer-Adsuara C, Hernandez-Bel L, MonteroHernandez J (2019) Intravitreal dexamethasone implant Ozurdex(R) in naive and refractory patients with different subtypes of diabetic macular edema. BMC Ophthalmol 19:15

18. Mello Filho P, Andrade G, Maia A, Maia M, Biccas Neto L, Muralha Neto A et al (2019) Effectiveness and Safety of Intravitreal Dexamethasone Implant (Ozurdex) in Patients with Diabetic Macular Edema: A Real-World Experience. Ophthalmologica 241:9-16

19. Allain H, Ramelet AA, Polard E, Bentue-Ferrer D (2004) Safety of calcium dobesilate in chronic venous disease, diabetic retinopathy and haemorrhoids. Drug Saf 27:649-660

20. Berthet P, Farine JC, Barras JP (1999) Calcium dobesilate: pharmacological profile related to its use in diabetic retinopathy. Int $\mathrm{J}$ Clin Pract 53:631-636

21. Liu J, Li S, Sun D (2019) Calcium Dobesilate and Micro-vascular diseases. Life Sci 221:348-353

22. Bogdanov P, Sola-Adell C, Hernandez C, Garcia-Ramirez M, Sampedro J, Simo-Servat O et al (2017) Calcium dobesilate prevents the oxidative stress and inflammation induced by diabetes in the retina of db/db mice. J Diabetes Complications 31:1481-1490

23. Ribeiro ML, Seres Al, Carneiro AM, Stur M, Zourdani A, Caillon P et al (2006) Effect of calcium dobesilate on progression of early diabetic retinopathy: a randomised double-blind study. Graefes Arch Clin Exp Ophthalmol 244:1591-1600

24. Akbulut B (2010) Calcium dobesilate and oxerutin: effectiveness of combination therapy. Phlebology $25: 66-71$

25. Photocoagulation for diabetic macular edema. Early Treatment Diabetic Retinopathy Study report number 1. Early Treatment Diabetic Retinopathy Study research group.Arch Ophthalmol103:17961806

26. James DGP, Mitkute D, Porter G, Vayalambrone D (2019) Visual Outcomes Following Intravitreal Ranibizumab for Diabetic Macular Edema in a Pro Re Nata Protocol from Baseline: A Real-World Experience. Asia Pac J Ophthalmol (Phila) 8:200-205

27. Haritoglou C, Gerss J, Sauerland C, Kampik A, Ulbig MW, group Cs (2009) Effect of calcium dobesilate on occurrence of diabetic macular oedema (CALDIRET study): randomised, double-blind, 
placebo-controlled, multicentre trial. Lancet 373:1364-1371

28. Zhang X, Liu W, Wu S, Jin J, Li W, Wang N (2015) Calcium dobesilate for diabetic retinopathy: a systematic review and meta-analysis. Sci China Life Sci 58:101-107

29. Graber R, Farine JC, Losa GA (1998) Calcium Dobesilate protects human peripheral blood mononuclear cells from oxidation and apoptosis. Apoptosis 3:41-49

30. Szabo ME, Haines D, Garay E, Chiavaroli C, Farine JC, Hannaert P et al (2001) Antioxidant properties of calcium dobesilate in ischemic/reperfused diabetic rat retina. Eur J Pharmacol 428:277-286

31. Rabe E, Jaeger KA, Bulitta M, Pannier F (2011) Calcium dobesilate in patients suffering from chronic venous insufficiency: a double-blind, placebo-controlled, clinical trial. Phlebology 26:162-168

32. Hall JF (2013) Modern management of hemorrhoidal disease. Gastroenterol Clin North Am 42:759772

33. Michal M, Gotti C (1988) Effect of calcium dobesilate on platelet function. Thromb Res 51:593-605

34. Brunet J, Farine JC, Garay RP, Hannaert P (1998) Angioprotective action of calcium dobesilate against reactive oxygen species-induced capillary permeability in the rat. Eur J Pharmacol 358:213220

35. Rota R, Chiavaroli C, Garay RP, Hannaert P (2004) Reduction of retinal albumin leakage by the antioxidant calcium dobesilate in streptozotocin-diabetic rats. Eur J Pharmacol 495:217-224

36. Zhou Y, Yuan J, Qi C, Shao X, Mou S, Ni Z (2017) Calcium dobesilate may alleviate diabetesinduced endothelial dysfunction and inflammation. Mol Med Rep 16:8635-8642

37. Zhou Y, Qi C, Li S, Shao X, Mou S, Ni Z (2018) Diabetic Nephropathy Can Be Treated with Calcium Dobesilate by Alleviating the Chronic Inflammatory State and Improving Endothelial Cell Function. Cell Physiol Biochem 51:1119-1133

38. Zhou Y, Qi C, Li S, Shao X, Ni Z (2019) Investigation of the Mechanism Underlying Calcium Dobesilate-Mediated Improvement of Endothelial Dysfunction and Inflammation Caused by High Glucose. Mediators Inflamm 2019:9893682

39. Angulo J, Peiro C, Romacho T, Fernandez A, Cuevas B, Gonzalez-Corrochano R et al (2011) Inhibition of vascular endothelial growth factor (VEGF)-induced endothelial proliferation, arterial relaxation, vascular permeability and angiogenesis by dobesilate. Eur J Pharmacol 667:153-159

40. Wang Y, Lu YH, Tang C, Xue M, Li XY, Chang YP et al (2019) Calcium Dobesilate Restores Autophagy by Inhibiting the VEGF/PI3K/AKT/mTOR Signaling Pathway. Front Pharmacol 10:886

41. Feghhi M, Farrahi F, Abbaspour M, Takhtaeian A (2014) Effect of adding oral calcium dobesilate to laser photocoagulation on the macular thickness in patients with diabetic macular edema: a randomized clinical trial. Adv Pharm Bull 4:375-378

42. Kim EJ, Lin WV, Rodriguez SM, Chen A, Loya A, Weng CY (2019) Treatment of Diabetic Macular Edema. Curr Diab Rep 19:68

43. Kodjikian L, Bellocq D, Bandello F, Loewenstein A, Chakravarthy U, Koh A et al (2019) First-line treatment algorithm and guidelines in center-involving diabetic macular edema. Eur $\mathrm{J}$ Ophthalmol 
29:573-584

44. Garcia Layana A, Adan A, Ascaso FJ, Cabrera F, Donate J, Escobar Barranco JJ et al (2020) Use of intravitreal dexamethasone implants in the treatment of diabetic macular edema: Expert recommendations using a Delphi approach. Eur J Ophthalmol 30:1042-1052

45. Mahapatra SK, Kumari S (2020) Long-term results of a single injection of intravitreal dexamethasone as initial therapy in diabetic macular edema. Indian J Ophthalmol 68:490-493

46. Njau F, Shushakova N, Schenk H, Wulfmeyer VC, Bollin R, Menne J et al (2020) Calcium dobesilate reduces VEGF signaling by interfering with heparan sulfate binding site and protects from vascular complications in diabetic mice. PLoS ONE 15:e0218494

\section{Figures}

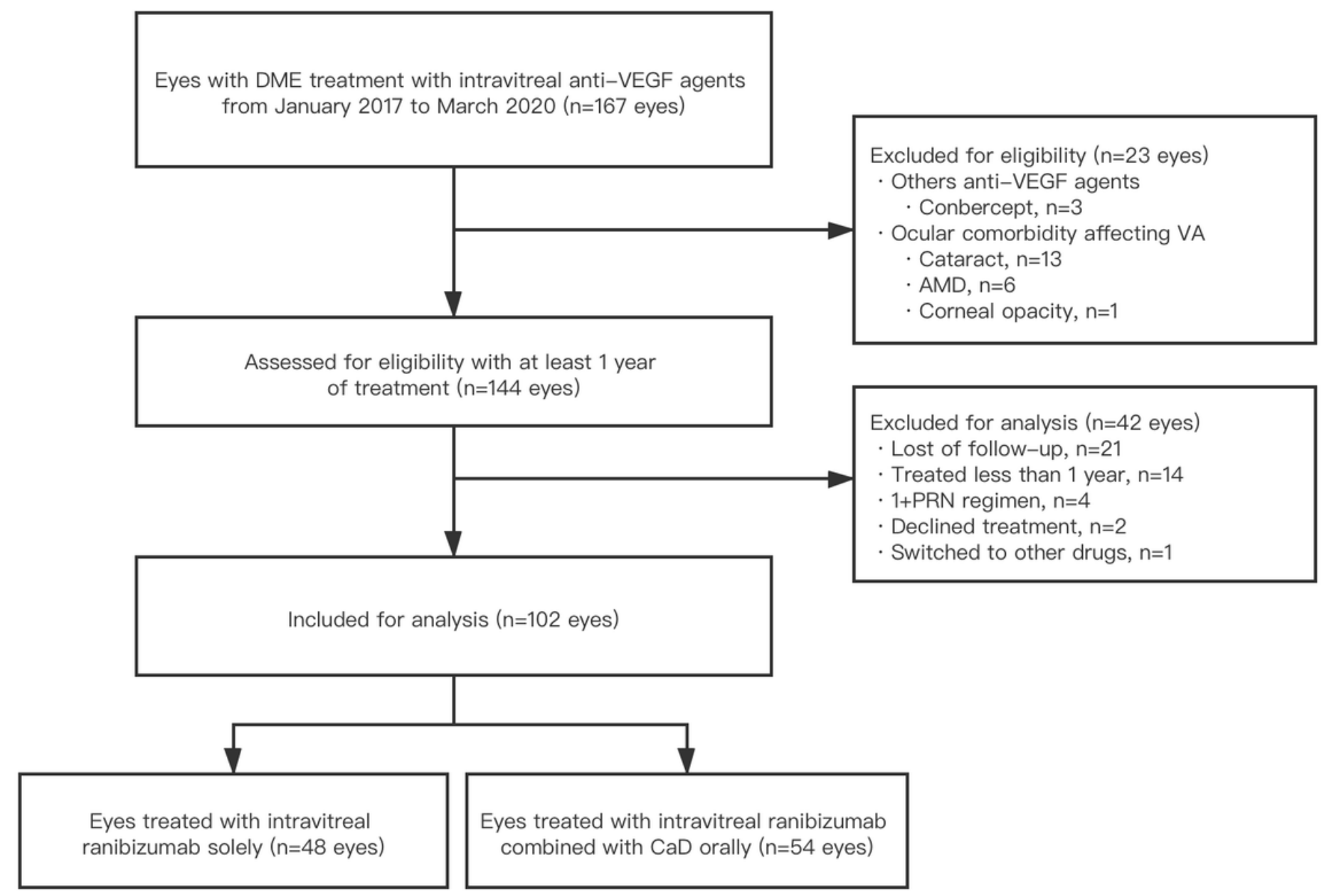

\section{Figure 1}

The flowchart indicated the protocol how to select the patients in this study. 